\title{
CD80 and CTLA-4 as diagnostic and prognostic markers in adult-onset minimal change disease: a retrospective study
}

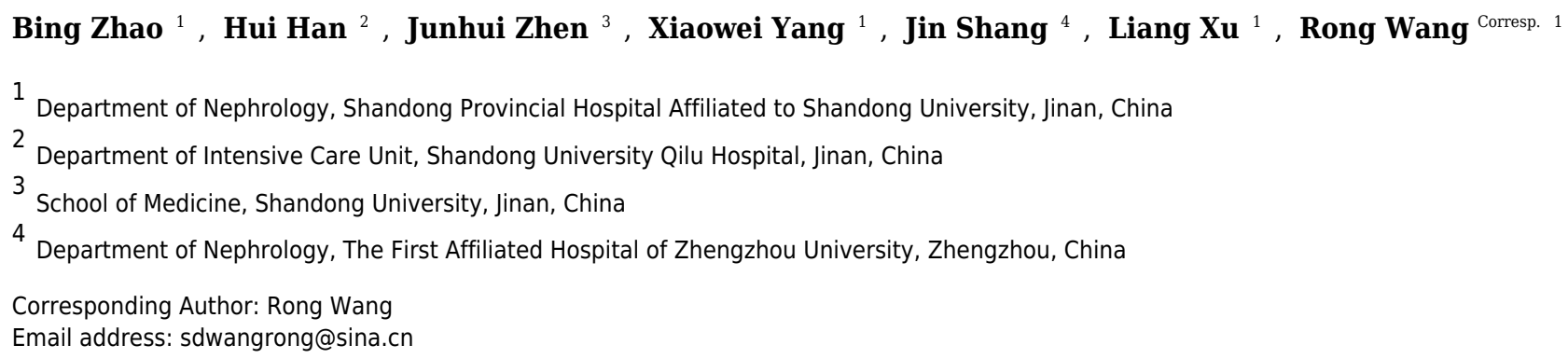

Background Minimal change disease (MCD) is a form of idiopathic nephrotic syndrome (INS). Compared to children, adult-onset MCD patients are reported to have delayed responses to glucocorticoid treatment. Several studies of children have suggested detecting urinary CD80 levels to diagnose MCD. There are no effective diagnostic method $s$ to distinguish steroid-sensitive MCD from steroid-resistant MCD unless treatments are used. Methods 55 patients with biopsy-proven MCD and 26 patients with biopsy-proven idiopathic membranous nephropathy (IMN), were detected CD80 and cytotoxic Tlymphocyte antigen-4 (CTLA-4) levels in serum, urine and renal tissue. Results Steroidsensitive MCD patients in remission had lower urinary CD80 levels and higher CTLA-4 levels than patients in relapse (156.65 \pm 24.62 vs $1066.40 \pm 176.76 \mathrm{ng} / \mathrm{g}$ creatinine; $p<0.0001)$, (728.73 \pm 89.93 vs $151.70 \pm 27.01 \mathrm{ng} / \mathrm{g}$ creatinine; $p<0.0001)$. For MCD patients in relapse, mean urinary CD80 level was higher, and CTLA-4 level was lower for those who were steroid-sensitive than those who were steroid-resistant (1066.40 \pm 176.76 vs $203.78 \pm 30.65 \mathrm{ng} / \mathrm{g}$ creatinine; $p<0.0001$ ), but the mean urinary CTLA-4 level was lower (151.70 \pm 27.01 vs $457.83 \pm 99.45 \mathrm{ng} / \mathrm{g}$ creatinine; $p<0.0001)$. CD80 expression in glomeruli was a sensitive marker to diagnose MCD. The absent or minimal expression of CTLA-4 in glomeruli could distinguish steroid-sensitive MCD from steroid-resistant MCD. Conclusions Glucocorticoid treatment may result in complete remission for only MCD patients with strongly positive CD80 expression and negative CTLA-4 expression in glomeruli, or higher urinary CD80 level and lower CTLA-4 level. 
1 CD80 and CTLA-4 as diagnostic and prognostic markers in adult-onset

2 minimal change disease: a retrospective study

3 Bing Zhao ${ }^{1, \#}$, Hui Han ${ }^{2, \#}$, Junhui Zhen ${ }^{3}$, Xiaowei Yang ${ }^{1}$, Jin Shang ${ }^{4}$, Liang Xu1 ${ }^{1}$, Rong Wang ${ }^{1}$ *

$4{ }^{1}$ Department of Nephrology, Shandong Provincial Hospital Affiliated to Shandong University,

5 Jinan, China.

$6{ }^{2}$ Department of Intensive Care Unit, Shandong University, Qilu Hospital, Jinan, China. ${ }^{3}$ School

7 of Medicine, Shandong University, Jinan, China.

$8{ }^{4}$ Department of Nephrology, the First Affiliated Hospital of Zhengzhou University, Zhengzhou, 9 China.

10

*Correspondence:

12 Prof. Rong Wang, Department of Nephrology, Shandong Provincial Hospital Affiliated to 13 Shandong University. No. 324, Jingwu Road, Jinan, China. Tel: +86 053168776458 ; E-mail: 14 sdwangrong@sina.cn.

15 \#Both authors contributed equally to this work. 
19 CD80 and CTLA-4 as diagnostic and prognostic markers in adult-onset minimal change disease: a retrospective study

\section{ABSTRACT}

\section{Background}

Minimal change disease (MCD) is a form of idiopathic nephrotic syndrome (INS). Compared to children, adult-onset MCD patients are reported to have delayed responses to glucocorticoid treatment. Several studies of children have suggested detecting urinary CD80 levels to diagnose MCD. There are no effective diagnostic methods to distinguish steroid-sensitive MCD from steroid-resistant MCD unless treatments are used.

\section{Methods}

We collected urine and serum samples from 55 patients with biopsy-proven MCD and 26 patients with biopsy-proven idiopathic membranous nephropathy (IMN) for testing CD80 and cytotoxic T-lymphocyte antigen-4 (CTLA-4) levels. A limited number of biopsies were available for detecting CD80 and CTLA-4, including 17 cases of MCD in relapse, 4 cases of MCD in remission, and 6 cases of IMN in relapse. In seven of eight MCD patients in relapse, Meanwhile, the laboratory examination of all the patients and the prognosis of MCD patients were recorded.

\section{Results}

Steroid-sensitive MCD patients in remission had lower urinary CD80 levels and higher CTLA-4 levels than patients in relapse ( $156.65 \pm 24.62$ vs $1066.40 \pm 176.76 \mathrm{ng} / \mathrm{g}$ creatinine; $p<0.0001)$, $(728.73 \pm 89.93$ vs $151.70 \pm 27.01 \mathrm{ng} / \mathrm{g}$ creatinine; $p<0.0001)$. For MCD patients in relapse, mean urinary CD80 level was higher, and CTLA-4 level was lower for those who were steroidsensitive than those who were steroid-resistant $(1066.40 \pm 176.76$ vs $203.78 \pm 30.65 \mathrm{ng} / \mathrm{g}$ creatinine; $p<0.0001)$, but the mean urinary CTLA-4 level was lower $(151.70 \pm 27.01$ vs 457.83 $\pm 99.45 \mathrm{ng} / \mathrm{g}$ creatinine; $p<0.0001)$. CD80 expression in glomeruli was a sensitive marker to diagnose MCD. The absent or minimal expression of CTLA-4 in glomeruli could distinguish steroid-sensitive MCD from steroid-resistant MCD.

\section{Conclusions}

Glucocorticoid treatment may result in complete remission for only MCD patients with strongly positive CD80 expression and negative CTLA-4 expression in glomeruli, or higher urinary CD80 level and lower CTLA-4 level. 
50

51

Minimal change disease (MCD) is a common form of idiopathic nephrotic syndrome (INS) that accounts for 10 to $15 \%$ of nephrotic diseases in adults ${ }^{1,2}$. MCD is a serious and challenging disease for adult-onset patients, of which around $25 \%$ are steroid-resistant. Compared to children, adult-onset MCD patients were reported to have higher risks of acute kidney injury and delayed responses to glucocorticoids treatment ${ }^{3,4}$. It has been reported that $73 \%$ of MCD patients experienced at least one relapse, of which $28 \%$ suffered frequent relapses ${ }^{2}$.

Nephrologists often put forward two options to MCD patients who relapse again: either receive the drug therapy ((i.e., full-dose glucocorticoid therapy, cytotoxic drugs), or seek further diagnostic tests, such as renal biopsy. Most patients tend to choose the former. These choices could result in ineffective treatments and/or increased adverse drug reactions ${ }^{5,6}$. The mechanism of steroid-resistant MCD is unknown. The characteristics of patients (e.g., age or medication compliance) and pathologic misdiagnosis, which may be due to similar imaging features of MCD, focal segmental glomerulosclerosis (FSGS), and stage I of idiopathic membranous nephropathy (IMN) under light microscopy are possible causes. Long-term prognosis may not be favorable, as indicated in a study in which a large number of patients with adult-onset MCD were found to have FSGS on a second kidney biopsy and experienced progression to end-stage renal disease (ESRD) or death ${ }^{4}$. According to the North American Pediatric Renal Trials and Collaborative Studies, steroid-resistant nephrotic syndrome constitutes the second most frequent cause of ESRD in the first 2 decades of life ${ }^{7}$. Therefore, early-stage identification of steroid-resistant nephritic syndrome is needed in MCD patients.

The pathogenesis of MCD remains unclear; however, several hypotheses have been proposed. For several decades, MCD has been considered a T-cell disorder, and increased levels of several cytokines were also suggested. Recently, a proposed "two-hit" theory proposed the induction of CD80 (B7-1) and regulatory T-cell dysfunction ${ }^{8}$, with or without impaired autoregulatory function of podocytes. Several studies of children suggested detecting urinary CD80 level to distinguish MCD from FSGS ${ }^{9-12}$, but there have been few studies of adult-onset MCD. Abatacept (cytotoxic T-lymphocyte-associated antigen 4-immunoglobulin fusion protein [CTLA-4-Ig]), a costimulatory inhibitor that targets CD80, has been used in CD80-accociated nephropathy ${ }^{13,14}$. However, its effectiveness is controversial ${ }^{13,15,16}$. Here, we aimed to evaluate whether CD80 and CTLA-4 could be diagnostic and prognostic markers in adult-onset MCD, and whether these markers could be useful for predicting the effectiveness of single- 
81 glucocorticoid treatment in adult-onset MCD patients.

82

83

MATERIALS AND METHODS

\section{Patients}

All patients were followed at Shandong Provincial Hospital affiliated to Shandong University. Patients over 14 years old are recommended by specialist physicians instead of pediatricians in China and receive diagnosis and treatment following standards for adults. The study was approved by the Institutional Review Board of Shandong Provincial Hospital affiliated to Shandong University (No.2014-022). Before participation in this study, written informed consent was obtained from all patients and their parents/guardians.

\section{Inclusion and exclusion criteria:}

Our research subjects were first selected from the hospitalized patients in the nephrology department of Shandong Provincial Hospital affiliated to Shandong University between March and November 2014.Our inclusion criteria were: (1) diagnosed as nephrotic syndrome patients by specialist physicians; (2) age no less than 14 years old; (3) renal pathological diagnoses as MCD or IMN; (4) eGFR calculated by Creatinine Equation ${ }^{17}$ higher than $60 \mathrm{ml} / \mathrm{min}$ per $1.73 \mathrm{~m}^{2}$. The exclusion criteria were: (1) pregnant women, tumor patients, and urinary system lithiasis patients; (2) lost to follow-up without any prognosis. The IMN patients were the control group. Definitions ${ }^{18,19,20}$

Complete Remission (CR): urinary protein excretion $<0.3 \mathrm{~g} / \mathrm{d}$ or urine protein:creatinine ratio $(\mathrm{uPCR})<30 \mathrm{mg} / \mathrm{mmol}$. Partial Remission: urinary protein excretion $<3.5 \mathrm{~g} / \mathrm{d}$ or $\mathrm{uPCR}<350$ $\mathrm{mg} / \mathrm{mmol}$ and a $50 \%$ or greater reduction from peak values, accompanied by an improvement or normalization of the serum albumin concentration. Relapse: proteinuria $>3.5 \mathrm{~g} / \mathrm{d}$ or $\mathrm{uPCR}>350$ $\mathrm{mg} / \mathrm{mmol}$. Steroid resistance: failure to achieve remission after 8 weeks of corticosteroid therapy. Steroid sensitivity: achieved CR during 8 weeks of corticosteroid therapy. Renal pathologic diagnoses of patients were established using light and electron microscopy by two pathologists. Glucocorticoid treatment: prednisone $1 \mathrm{mg} / \mathrm{kg}$ per day for 8 weeks, and then reduce the $10 \%$ of total dosage every two weeks.

\section{CD80 and CTLA-4 measurements}

Serum and urinary CD80 and CTLA-4 were detected when patients were in relapse or complete/partial remission, and 24-hour urinary protein, uPCR and serum albumin were measured in the same day. CD80 and CTLA-4 levels were measured using a commercially 
113 available ELISA kit (Bender MedSystems, eBioscience, Vienna, Austria), and results were

114 adjusted for urinary creatinine excretion. Urinary creatinine level and protein and serum albumin

115 levels were measured using an autoanalyzer.

\section{Immunohistochemistry}

117 Kidney samples were obtained from excess tissue corresponding to kidney nephrectomy

118

119

120

121

122

123

124

125

126

127

128

129

130

131

132

133

134

135

136

137

138

139

140

141

142

143

specimens donated to the biobank of Shandong University after diagnostic evaluation.

Immunohistochemistry involved 5- $\mu$ m-thick paraffin-embedded tissue sections. The primary antibodies were mouse anti-human monoclonal CD80 (1:150) and CTLA-4 (1:100, both Santa Cruz Biotechnology), and the secondary antibodies were a rabbit anti-mouse Biotin-Streptavidin HRP Detection Systems (Zhongshanjinqiao Biotechnology company, Beijing, China). Sections were counterstained with Carazzi's hematoxylin.

\section{Statistical analysis}

Statistical analyses were performed with SPSS 17.0, and receiver operating characteristic (ROC) curve analyses were performed with Medcalc 17.0. Data were analyzed by $t$-test. Results were considered significant at $p<0.05$.

\section{RESULTS}

We detected serum and urinary CD80 and CTLA-4 levels by ELISA in 55 patients with biopsyproven MCD and 26 patients with biopsy-proven idiopathic membranous nephropathy (IMN).

Comparison of urinary CD80 and CTLA-4 excretion of steroid-sensitive MCD patients in relapse and remission

We detected all the laboratory data (levels of serum albumin, 24-hour urinary protein, serum CD80 and CTLA-4, urinary CD80 and CTLA-4) for all our steroid-sensitive MCD patients when they were in relapse and remission. The laboratory data and glucocorticoid treatments are shown in Table 1.

Urinary CD80 excretion was lower for MCD patients in remission than relapse (156.65 \pm 24.62 vs $1066.40 \pm 176.76 \mathrm{ng} / \mathrm{g}$ creatinine; $p<0.0001$ ). However, mean urinary CTLA-4 levels were greater for MCD patients in remission than relapse $(728.73 \pm 89.93$ vs $151.70 \pm 27.01 \mathrm{ng} / \mathrm{g}$ creatinine; $p<0.0001$ ) (Table 1 and Figure 1).

The area under the receiver operating characteristic curve (AUC) comparing MCD patients in relapse versus remission was 0.957 for urinary CD80 and 0.0.928 for urinary CTLA-4 (Figure 2), with no significant difference between these two AUCs. 
The serum CD80 and CTLA-4 levels of steroid-sensitive MCD patients in relapse were not statistically different from these patients when they were in remission (Table 1).

\section{Comparison of urinary CD80 and CTLA-4 excretion of steroid-sensitive MCD patients in} relapse, steroid-resistant MCD patients in relapse and IMN in relapse

We compared the CD80 and CTLA-4 levels of 32 steroid-sensitive MCD patients in relapse, 23 steroid-resistant MCD patients in relapse, and 26 IMN patients in relapse. The laboratory data and glucocorticoid treatments are shown in Table 2.

The urinary CD80 level of steroid-sensitive MCD patients in relapse was significantly higher than that of steroid-sensitive MCD patients in relapse (1066.40 \pm 176.76 vs $203.78 \pm 30.65$ $\mathrm{ng} / \mathrm{g}$ creatinine; $p<0.0001$ ), and it was also significantly higher than that of IMN patients in relapse $(1066.40 \pm 176.76$ vs $294.95 \pm 34.08 \mathrm{ng} / \mathrm{g}$ creatinine; $p<0.0001)$. Urinary CTLA-4 levels of steroid-sensitive MCD patients in relapse were significantly lower than those of steroidsensitive MCD patients in relapse $(151.70 \pm 27.01$ vs $457.83 \pm 99.45 \mathrm{ng} / \mathrm{g}$ creatinine; $p=0.006)$, and they were also significantly lower than those of IMN patients in relapse $(151.70 \pm 27.01 \mathrm{vs}$ $299.53 \pm 47.46 \mathrm{ng} / \mathrm{g}$ creatinine; $p=0.006$ ). The urinary CD80 levels of steroid-resistant MCD patients in relapse was not different statistically from IMN patients in relapse, as were the urinary CTLA-4 levels of steroid-resistant MCD patients in relapse (Figure 3). Serum CD80 or CTLA-4 levels did not differ among groups. In comparing steroid-sensitive and steroid-resistant MCD patients in relapse, the AUC for urinary CD80 level was 0.937, and for urinary CTLA-4, it was 0.736 (Figure 4a). In comparing steroid-sensitive MCD patients and IMN patients in relapse, the AUC for urinary CD80 was 0.867 , and for urinary CTLA-4, it was 0.721 (Figure 4b).

\section{CD80 expressed in glomeruli in steroid-sensitive MCD patients in relapse}

A limited number of biopsies were available for study, including 17 cases of MCD in relapse, 4 cases of MCD in remission, and 6 cases of IMN in relapse. The remission in the 4 cases of MCD was partial (proteinuria $<1 \mathrm{~g} / 24 \mathrm{~h}$ ) at the time of renal biopsy, and several days after biopsy, all 4 cases showed CR. Glucocorticoids or other immunosuppressive drugs had been used in the IMN cases.

CD80 was present in the glomeruli of patients with steroid-sensitive MCD in relapse, but was minimal or absent for those with steroid-sensitive MCD in remission. CTLA-4 was minimal or absent in the glomeruli of patients with steroid-sensitive MCD in relapse, but was present in the glomeruli of those with steroid-sensitive MCD in remission. Both CD80 and CTLA-4 were 
175 present in the glomeruli of patients with steroid-resistant MCD and IMN in relapse, and levels 176 were minimal (Figure 5).

\section{DISCUSSION}

178 Few studies have investigated the expression of CD80 and CTLA-4 in adult-onset MCD. Our 179 study demonstrated that urinary CD80 was elevated in MCD during relapse, with levels returning 180 to the low range with disease in remission. Urinary CTLA-4 levels were higher in patients in 181 remission than relapse.

182 Some studies have investigated these levels in children ${ }^{10,11}$. However, patients with primary 183 adult-onset MCD may have more severe clinical features than pediatric MCD patients. A recent study showed that only $30 \%$ of adult Chinese MCD patients achieved CR after initial treatment ${ }^{4}$. In addition, the long-term prognosis may not be favorable, as indicated in a study finding that a considerable number of patients with adult-onset MCD, showing FSGS on a second kidney biopsy who experienced progression to ESRD or death ${ }^{4}$. Some studies of patients with adultonset MCD have reported increased risk of acute kidney injury ${ }^{3,4}$ and delayed response to treatment with glucocorticoid therapy as compared to pediatric MCD patients ${ }^{2,21}$. In our study, the variation in urinary CD80 levels in steroid-sensitive adult-onset MCD was similar to that for pediatric MCD patients. Urinary CD80 and CTLA-4 levels seem to be associated with MCD activity in adults.

We found no differences in serum CD80 or CTLA-4 level in patients in remission than those in relapse; thus, the increased urinary excretion could not be explained by increased serum levels. The immunohistochemical expression of CD80 or CTLA-4 on renal tissue paralleled changes in urinary CD80 or CTLA-4 excretion from relapse to partial remission for steroid-sensitive MCD patients. Urinary CD80 or CTLA-4 may be excreted from the kidney but not blood circulation. Garin et al. tested whether the source of urinary CD80 is podocytes because of differences in molecular weight between soluble CD80 secreted by circulating B cells and whole-cell membrane-associated CD80 $0^{22,23}$ and with immunofluorescence studies of renal biopsies ${ }^{11}$.

CD80, also termed B7-1, is a transmembrane protein expressed on the surface of B cells and other antigen-presenting cells. It is one of the major co-stimulators of T-cell activation by binding to its counter-receptors CD28 and CTLA-4. CD80 can be induced by the endotoxin lipopolysaccharide via toll-like receptor 4 activation. Lipopolysaccharide (LPS) injection leads to transient podocyte foot-process effacement and proteinuria in mice, which is independent of 
206 lymphocytes because it also occurs in SCID mice, which are devoid of lymphocytes. However, 207 the LPS model is self-limiting as compared with the prolonged course of MCD in humans ${ }^{24,25}$. 208 The mechanisms that promote persistent CD80 expression in MCD remain unknown. Reiser et $a l .{ }^{26}$ speculated that CD80 induction in podocytes may be a physiological response to infection and facilitate the excretion of pathogens by transiently increasing the glomerular permeability to macromolecules. Modifying genetic or environmental factors may lead to persistent CD80 induction after a triggering event in MCD. Garin et al. ${ }^{10}$ postulated that MCD may be due in part to a defect in the ability of the immune system to turn off podocyte CD80 expression. Previous studies have suggested that soluble CTLA-4 produced by regulatory T cells can bind to dendritic cells expressing CD80 and act to block T-cell activation ${ }^{27}$. Garin et al. postulated that ineffective censoring of CD80 expression by T-regulatory cells may underlie the pathogenesis of MCD in light of the lower serum and urinary level of CTLA-4 in MCD patients in relapse ${ }^{10}$. However, we found higher CTLA-4 levels in the serum of MCD patients in relapse versus remission, although these differences were not significant. In our study, CTLA-4 was absent in renal biopsies of patients in relapse, but present in those with partial remission. CTLA-4, which can turn off podocyte CD80 expression, may arise from renal tissue instead of blood circulation.

Both urinary CD80 and CTLA-4 levels differed between patients with steroid-sensitive and resistant MCD in relapse. The urinary CD80 level was lower in patients with steroid-resistant than -sensitive MCD, but did not significantly differ from the urinary CD80 levels in patients with IMN in relapse. When urinary CD80 levels are significantly higher and urinary CTLA-4 levels are lower, glucocorticoids therapy may achieve CR.

CD80 was still present in the glomeruli of patients with steroid-resistant MCD. Serum CD80 levels did not differ between patients with steroid-sensitive and -resistant MCD. However, CTLA-4 was present in both glomeruli and urine.

In comparing patients in relapse with steroid-resistant MCD and IMN, we found no significant differences in serum or urinary CD80 or CTLA-4 levels in renal tissues. Some IMN cases were steroid-resistant. CTLA-4 may fail to turn off some podocyte CD80 expression and therefore result in complete CD80 excretion to urine even with full-dose glucocorticoid therapy.

Early changes in gene expression could affect the course of primary glomerular disease ${ }^{28}$. A decrease in expression of podocyte protein-tyrosine phosphatase (GLEPP1) was associated with partial steroid sensitivity in several mouse models of podocyte disease, but, in contrast to 
237 GLEPP1, upregulated CD80 expression is steroid-resistant. Recent studies have found that CD80

238 is expressed in renal tissue in several types of glomerulonephritis, such as lupus nephritis, 239 immunoglobulin A (IgA) nephropathy, diabetic nephropathy, and Fabry disease 24,29-32, in 240 addition to MCD. B7/CD28 blockade (LEA29Y, Belatacept) in kidney transplant recipients have proven 241 that the replacement of toxic CNI use is feasible in selected populations. ${ }^{33}$ Abatacept (CTLA-4-Ig fusion 242 protein), a costimulatory inhibitor that targets CD80, induced partial or complete remission of 243 proteinuria in patients with FSGS ${ }^{13,14}$, in which CD80 seemed to be minimally expressed in 244 glomeruli and urine ${ }^{11}$. However, few of these studies showed high urinary CD80 excretion in various nephritis diseases except MCD.

246 The method by which CD80 occurs in urine remains unknown. Previous studies have 247 speculated that urinary CD80 presence may not reflect that CD80-positive podocytes are lost in 248 the urine ${ }^{30,34,35}$. CD80 may be contained in granular membrane structures found in urine during 249 podocyte injury ${ }^{36}$. Some studies have found that slit diaphragm proteins are shed into the 250 urine ${ }^{37,38}$. CD80 that binds and sequesters slit diaphragm proteins may follow these proteins that 251 are shed ${ }^{24}$. Why CD80 cannot be completely shed from podocytes in steroid-resistant MCD or 252 other nephritis diseases, as in steroid-sensitive MCD, remains elusive. According to our data, 253 dysfunction of CTLA-4 may play an important role in the pathogenesis of MCD, shedding light 254 on further nephrotic research.

255 Nonetheless, we suggest that CD80 expression in renal tissue cannot be used to distinguish MCD from other nephritis diseases and cannot distinguish steroid-sensitive from -resistant MCD.

257 However, CD80+/CTLA-4- expression on glomeruli may indicate the functional deficiency of T258 regulatory cells ${ }^{39}$. Because of strongly positive expression in MCD patients and simultaneous negative CTLA-4 expression, glucocorticoid treatment might be effective. Increased urinary CD80 levels and reduced urinary CTLA-4 levels show a higher accessibility to remission and better sensitivity of full-dose glucocorticoid therapy. Our study helps accelerate adult MCD therapy for at least 8 weeks by allowing doctors to prescribe immune depressive drugs together with glucocorticoids based on our proposed CD80 and CTLA-4 levels.

In conclusion, for patients with MCD, strongly positive CD80 expression and simultaneous negative CTLA-4 expression, or higher urinary CD80 level and lower urinary CTLA-4 level, 
268 play a role in diagnosis and prognosis as non-invasive biomarkers. Further studies investigating 269 the precise mechanisms of the interaction of CD80 and CTLA-4 during the whole course of 270 MCD are needed.

271 ABBREVIATIONS

272 CTLA-4: cytotoxic T-lymphocyte antigen-4

273 MCD: minimal change disease

274 INS: idiopathic nephrotic syndrome

275 IMN: idiopathic membranous nephropathy

276 FSGS: focal segmental glomerulosclerosis

277 ESRD: end-stage renal disease

278 CR: complete remission

279 UPCR: urine protein:creatinine ratio

280 LPS: lipopolysaccharide

281 SCID: severe combined immune deficiency

282 ROC: receiver operating characteristic

283 DECLARATIONS

284 Consent to publish

285 Not applicable.

286 Acknowledgments

287 The authors thank all patients who participated in this study and the nurses from the nephrology 288 department of Shandong Provincial Hospital affiliated to Shandong University.

289 REFERRENCES

2901 Zech P, Colon S, Pointet P, Deteix P, Labeeuw M, and Leitienne P. 1982. The nephrotic 291 syndrome in adults aged over 60: etiology, evolution and treatment of 76 cases. Clin $292 \quad$ Nephrol 17:232-236.

2932 Waldman M, Crew RJ, Valeri A, Busch J, Stokes B, Markowitz G, D'Agati V, and Appel 294 G. 2007. Adult minimal-change disease: clinical characteristics, treatment, and outcomes. $295 \quad$ Clin J Am Soc Nephrol 2:445-453. 10.2215/cjn.03531006.

2963 Huang JJ, Hsu SC, Chen FF, Sung JM, Tseng CC, and Wang MC. 2001. Adult-onset 297 minimal change disease among Taiwanese: clinical features, therapeutic response, and prognosis. Am J Nephrol 21:28-34. 46215. 
2994 Szeto CC, Lai FM, Chow KM, Kwan BC, Kwong VW, Leung CB, and Li PK. 2015. 300 Long-term outcome of biopsy-proven minimal change nephropathy in Chinese adults. $\mathrm{Am}$

301

3025

303

304

305

306

307

308

309

310

311

312

313

314

315

316

317 J Kidney Dis 65:710-718. 10.1053/j.ajkd.2014.09.022.

5 Barbarino JM, Staatz CE, Venkataramanan R, Klein TE, and Altman RB. 2013. PharmGKB summary: cyclosporine and tacrolimus pathways. Pharmacogenet Genomics 23:563-585. 10.1097/FPC.0b013e328364db84.

6 Grimm M, Rinaldi M, Yonan NA, Arpesella G, Arizon Del Prado JM, Pulpon LA, Villemot JP, Frigerio M, Rodriguez Lambert JL, Crespo-Leiro MG, Almenar L, Duveau D, Ordonez-Fernandez A, Gandjbakhch J, Maccherini M, and Laufer G. 2006. Superior prevention of acute rejection by tacrolimus vs. cyclosporine in heart transplant recipients-a large European trial. $A m \quad J$ Transplant 6:1387-1397. 10.1111/j.16006143.2006.01300.x.

7 Smith JM, Martz K, and Blydt-Hansen TD. 2013. Pediatric kidney transplant practice patterns and outcome benchmarks, 1987-2010: a report of the North American Pediatric Renal Trials and Collaborative Studies. Pediatr Transplant 17:149-157. 10.1111/petr.12034.

8 Shimada M, Araya C, Rivard C, Ishimoto T, Johnson RJ, and Garin EH. 2011. Minimal change disease: a "two-hit" podocyte immune disorder? Pediatr Nephrol 26:645-649. 10.1007/s00467-010-1676-x.

Cara-Fuentes G, Wei C, Segarra A, Ishimoto T, Rivard C, Johnson RJ, Reiser J, and Garin EH. 2014b. CD80 and suPAR in patients with minimal change disease and focal segmental glomerulosclerosis: diagnostic and pathogenic significance. Pediatr Nephrol 29:1363-1371. 10.1007/s00467-013-2679-1.

10 Garin EH, Diaz LN, Mu W, Wasserfall C, Araya C, Segal M, and Johnson RJ. 2009. Urinary CD80 excretion increases in idiopathic minimal-change disease. $J \mathrm{Am} \mathrm{Soc}$ Nephrol 20:260-266. 10.1681/asn.2007080836.

1 Garin EH, Mu W, Arthur JM, Rivard CJ, Araya CE, Shimada M, and Johnson RJ. 2010. Urinary CD80 is elevated in minimal change disease but not in focal segmental glomerulosclerosis. Kidney Int 78:296-302. 10.1038/ki.2010.143.

12 Ling C, Liu X, Shen Y, Chen Z, Fan J, Jiang Y, and Meng Q. 2015. Urinary CD80 levels as a diagnostic biomarker of minimal change disease. Pediatr Nephrol 30:309-316. 
330

$331 \quad 13$

332

333

334

335

336

337

338

339

340

341

$342 \quad 16$

343

344

345

346

347

348

349

350

351

352

353

354

355

356

357

358

359

360

10.1007/s00467-014-2915-3.

13 Yu CC, Fornoni A, Weins A, Hakroush S, Maiguel D, Sageshima J, Chen L, Ciancio G, Faridi MH, Behr D, Campbell KN, Chang JM, Chen HC, Oh J, Faul C, Arnaout MA, Fiorina P, Gupta V, Greka A, Burke GW, 3rd, and Mundel P. 2013. Abatacept in B7-1positive proteinuric kidney disease. $N$ Engl $J$ Med 369:2416-2423. 10.1056/NEJMoa1304572.

14 Trimarchi H. 2015. Abatacept and Glomerular Diseases: The Open Road for the Second Signal as a New Target is Settled Down. Recent Pat Endocr Metab Immune Drug Discov 9:2-14.

15 Norlin J, Nielsen Fink L, Helding Kvist P, Douglas Galsgaard E, and Coppieters K. 2016. Abatacept Treatment Does Not Preserve Renal Function in the Streptozocin-Induced Model of Diabetic Nephropathy. PLoS One 11:e0152315. 10.1371/journal.pone.0152315. arin EH, Reiser J, Cara-Fuentes G, Wei C, Matar D, Wang H, Alachkar N, and Johnson RJ. 2015. Case series: CTLA4-IgG1 therapy in minimal change disease and focal segmental glomerulosclerosis. Pediatr Nephrol 30:469-477. 10.1007/s00467-014-2957-6.

17 Andrew S. Levey, Lesley A. Stevens, Christopher H. Schmid, Yaping (Lucy) Zhang, Alejandro F. Castro III, Harold I. Feldman, John W. Kusek, Paul Eggers, Frederick Van Lente, Tom Greene, PhD6, and Josef Coresh. 2009.: A new equation to estimate glomerular filtration rate. Ann Intern Med 150(9):604-612.

18 Kidney Disease: Improving Global Outcomes (KDIGO) Glomerulonephritis Work Group. 2012. KDIGO Clinical Practice Guideline for Glomerulonephritis. Kidney Int Suppl 2: $139-274$.

19 Meyers KEC, Kaplan BS. 2001. Immunologic renal diseases. 2nd ed. Philadelphia: Lippincott Williams \& Wilkins.

20. Haiyan Wang. 2009. Nephrology. 3rd. Beijing: People's Medical Publishing House.

21 Korbet SM, Schwartz MM, and Lewis EJ. 1988. Minimal-change glomerulopathy of adulthood. Am J Nephrol 8:291-297.

22 Greenwald RJ, Freeman GJ, and Sharpe AH. 2005. The B7 family revisited. Annu Rev Immunol 23:515-548. 10.1146/annurev.immunol.23.021704.115611.

23 Wong CK, Lit LC, Tam LS, Li EK, and Lam CW. 2005. Aberrant production of soluble costimulatory molecules CTLA-4, CD28, CD80 and CD86 in patients with systemic 
361

$362 \quad 24$

363

364

365

366

367

368

369

370

371

372

373

374

375

376

377

378

379

380

381

382

383

384

385

386

$387 \quad 31$

388

389

$390 \quad 32$

lupus erythematosus. Rheumatology (Oxford) 44:989-994. 10.1093/rheumatology/keh663.

Reiser J, von Gersdorff G, Loos M, Oh J, Asanuma K, Giardino L, Rastaldi MP, Calvaresi N, Watanabe H, Schwarz K, Faul C, Kretzler M, Davidson A, Sugimoto H, Kalluri R, Sharpe AH, Kreidberg JA, and Mundel P. 2004. Induction of B7-1 in podocytes is associated with nephrotic syndrome. J Clin Invest 113:1390-1397. 10.1172/jci20402.

25 Kistler AD, and Reiser J. 2010. Maximal 'CD80-uria' with minimal change. Kidney Int 78:236-238. 10.1038/ki.2010.148.

26 Reiser J, and Mundel P. 2004. Danger signaling by glomerular podocytes defines a novel function of inducible B7-1 in the pathogenesis of nephrotic syndrome. J Am Soc Nephrol 15:2246-2248. 10.1097/01.asn.0000136312.46464.33.

27 Taylor PA, Lees CJ, Fournier S, Allison JP, Sharpe AH, and Blazar BR. 2004. B7 expression on $\mathrm{T}$ cells down-regulates immune responses through CTLA-4 ligation via TT interactions [corrections]. J Immunol 172:34-39.

28 Clement LC, Liu G, Perez-Torres I, Kanwar YS, Avila-Casado C, and Chugh SS. 2007. Early changes in gene expression that influence the course of primary glomerular disease. Kidney Int 72:337-347. 10.1038/sj.ki.5002302.

29 Fiorina P, Vergani A, Bassi R, Niewczas MA, Altintas MM, Pezzolesi MG, D'Addio F, Chin M, Tezza S, Ben Nasr M, Mattinzoli D, Ikehata M, Corradi D, Schumacher V, Buvall L, Yu CC, Chang JM, La Rosa S, Finzi G, Solini A, Vincenti F, Rastaldi MP, Reiser J, Krolewski AS, Mundel PH, and Sayegh MH. 2014. Role of podocyte B7-1 in diabetic nephropathy. J Am Soc Nephrol 25:1415-1429. 10.1681/asn.2013050518.

30 Trimarchi H, Canzonieri R, Schiel A, Costales-Collaguazo C, Politei J, Stern A, Paulero M, Rengel T, Andrews J, Forrester M, Lombi M, Pomeranz V, Iriarte R, Muryan A, Zotta E, Sanchez-Nino MD, and Ortiz A. 2016. Increased urinary CD80 excretion and podocyturia in Fabry disease. $J$ Transl Med 14:289. 10.1186/s12967-016-1049-8.

$31 \mathrm{Wu}$ Q, Jinde K, Endoh M, and Sakai H. 2004. Clinical significance of costimulatory molecules CD80/CD86 expression in IgA nephropathy. Kidney Int 65:888-896. 10.1111/j.1523-1755.2004.00477.x.

32 Sui MS, Zhou J, Jia XB, Mu SH, Liu XG, Ji Y, and Xie RJ. 2010. [Expression and 
391

392

393

394

395

396

397

398

399

400

401

402

$403 \quad 36$

404

405

$406 \quad 37$

407

408

409

$410 \quad 38$

411

412

413

414

415

416

417

418

419

420

significance of CD80/CD86 in renal tissue of lupus nephritis]. Zhonghua Nei Ke Za Zhi 49:691-695.

33 D'Addio F, Boenisch O, Magee CN, Yeung MY, Yuan X, Mfarrej B, Vergani A, Ansari MJ, Fiorina P, and Najafian N. 2013. Prolonged, low-dose anti-thymocyte globulin, combined with CTLA4-Ig, promotes engraftment in a stringent transplant model. PLoS One 8:e53797. 10.1371/journal.pone.0053797.

34 Petermann A, and Floege J. 2007. Podocyte damage resulting in podocyturia: a potential diagnostic marker to assess glomerular disease activity. Nephron Clin Pract 106:c61-66. 10.1159/000101799.

35 Yu D, Petermann A, Kunter U, Rong S, Shankland SJ, and Floege J. 2005. Urinary podocyte loss is a more specific marker of ongoing glomerular damage than proteinuria. $J$ Am Soc Nephrol 16:1733-1741. 10.1681/asn.2005020159.

36 Hara M, Yanagihara T, Kihara I, Higashi K, Fujimoto K, and Kajita T. 2005. Apical cell membranes are shed into urine from injured podocytes: a novel phenomenon of podocyte injury. J Am Soc Nephrol 16:408-416. 10.1681/asn.2004070564.

37 Gerke P, Sellin L, Kretz O, Petraschka D, Zentgraf H, Benzing T, and Walz G. 2005. $\mathrm{NEPH} 2$ is located at the glomerular slit diaphragm, interacts with nephrin and is cleaved from podocytes by metalloproteinases. $J$ Am Soc Nephrol 16:1693-1702. 10.1681/asn.2004060439.

38 Collino F, Bussolati B, Gerbaudo E, Marozio L, Pelissetto S, Benedetto C, and Camussi G. 2008. Preeclamptic sera induce nephrin shedding from podocytes through endothelin1 release by endothelial glomerular cells. Am J Physiol Renal Physiol 294:F1185-1194. 10.1152/ajprenal.00442.2007.

39 Cara-Fuentes G, Wasserfall CH, Wang H, Johnson RJ, and Garin EH. 2014a. Minimal change disease: a dysregulation of the podocyte CD80-CTLA-4 axis? Pediatr Nephrol 29:2333-2340. 10.1007/s00467-014-2874-8.

\section{Table legends}

Table 1. Laboratory data of steroid-sensitive MCD patients in remission and relapse.

Table 2. Laboratory data and therapy for steroid-sensitive MCD patients in relapse, 
421 steroid-resistant MCD patients in relapse, and IMN patients in relapse.

422 Figure legends

423 Figure 1. Urinary CD80 and urinary CTLA-4 levels of steroid-sensitive MCD patients 424 in relapse and remission.

425 Figure 2. Receiver operating characteristic curves for differentiating relapse and 426 remission in patients with steroid-sensitive MCD.

Figure 3. Comparison of urinary CD80 and CTLA-4 levels of steroid-sensitive MCD patients in relapse, steroid-resistant MCD patients in relapse, and IMN patients in relapse.

Figure 4. Receiver operating characteristic curves (ROC) for urinary CD80 with CTLA-4 levels differentiating patients with steroid-sensitive MCD and others. ROC analysis of urinary CD80 with CTLA-4 levels comparing steroid-sensitive MCD and steroidresistant MCD patients in relapse (A) and steroid-sensitive MCD and IMN patients in relapse (B). Figure 5. Expression of CD80 and CTLA-4 in the glomerulus of several types of idiopathic nephrotic syndrome. (A, E, and G) CD80 was expressed (brown stain) in the glomerulus from a steroid-sensitive MCD patient in relapse, a steroid-resistant MCD patient in relapse, and an IMN patient in relapse. (C) Minimal stain for CD80 was found in the glomerulus of a steroid-sensitive MCD in partial remission. (D, F, and H) CTLA-4 was expressed in the glomerulus from the steroid-resistant MCD patient in remission, the steroid-resistant MCD patient in relapse, and an IMN patient in relapse. (B) CTLA-4 was absent in the glomerulus from the steroid-sensitive MCD patient in relapse. (Immunohistochemistry, original magnification $\times 200$ ) 


\section{Table 1 (on next page)}

Laboratory data of steroid-sensitive MCD patients in remission and relapse 
1 Table 1. Laboratory data of steroid-sensitive MCD patients in remission and relapse

\begin{tabular}{ccc}
\hline Laboratory data & Relapse & Remission \\
\hline Serum albumin $(\mathrm{g} / \mathrm{l})$ & $20.04 \pm 1.19^{\mathrm{a}}$ & $27.83 \pm 1.19$ \\
24-hour urinary protein $(\mathrm{g})$ & $5.33 \pm 0.35$ & $0.34 \pm 0.09$ \\
Urinary CD80 $(\mathrm{ng} / \mathrm{g}$ creat $)$ & $1066.40 \pm 176.76$ & $156.65 \pm 24.62$ \\
$\left(95 \% \mathrm{CI}^{\mathrm{b}}\right)$ & $(705.90-1426.91)$ & $(106.44-206.86)$ \\
Urinary CTLA-4 $(\mathrm{ng} / \mathrm{g}$ creat $)$ & $151.70 \pm 27.01$ & $728.73 \pm 89.93$ \\
$(95 \% \mathrm{CI})$ & $(96.61-206.79)$ & $(545.33-912.13)$ \\
Serum CD $80(\mathrm{ng} / \mathrm{l})$ & $0.87 \pm 0.12$ & $0.63 \pm 0.08$ \\
$(95 \% \mathrm{CI})$ & $(0.63-1.11)$ & $(0.47-0.79)$ \\
Serum CTLA-4 (ng/l) & $0.33 \pm 0.07$ & $0.33 \pm 0.07$ \\
$(95 \% \mathrm{CI})$ & $(0.19-0.47)$ & $(0.19-0.46)$ \\
Glucocorticoids treatment & & 11 \\
None & 19 & 16 \\
1-7 days & 9 & 2 \\
8-28 days & 1 & 3 \\
\hline 29-112 days & 0 & \\
\hline
\end{tabular}

${ }^{a}$ Data are mean \pm s.e.m

${ }^{\mathrm{b}} \mathrm{CI}$ : Confidence Interval for mean

2 
Table 2 (on next page)

Laboratory data and therapy for steroid-sensitive MCD patients in relapse, steroidresistant MCD patients in relapse and IMN in relapse 
1 Table 2. Laboratory data and therapy for steroid-sensitive MCD patients in relapse,

2 steroid-resistant MCD patients in relapse and IMN in relapse

\begin{tabular}{|c|c|c|c|}
\hline Laboratory data & $\begin{array}{l}\text { Steroid-sensitive } \\
\text { MCNS patients in } \\
\text { relapse }\end{array}$ & $\begin{array}{l}\text { Steroid-resistant } \\
\text { MCNS patients in } \\
\text { relapse }\end{array}$ & $\begin{array}{l}\text { IMN patients in } \\
\text { relapse }\end{array}$ \\
\hline Age $(\mathrm{yr})($ mean $\pm \mathrm{SD})$ & $27.63 \pm 12.55$ & $33.09 \pm 17.99$ & $38.77 \pm 14.63$ \\
\hline Serum albumin $(\mathrm{g} / \mathrm{l})$ & $20.04 \pm 1.19^{\mathrm{a}}$ & $21.85 \pm 1.70$ & $25.91 \pm 1.21$ \\
\hline 24-hr urinary protein $(\mathrm{g})$ & $5.33 \pm 2.00$ & $4.78 \pm 0.38$ & $4.45 \pm 0.38$ \\
\hline Urinary CD80 (ng/g creat) & $1066.40 \pm 176.76$ & $203.78 \pm 30.65$ & $294.95 \pm 34.08$ \\
\hline$\left(95 \% \mathrm{CI}^{\mathrm{b}}\right)$ & $(705.90-1426.91)$ & $(140.21-267.34)$ & $(224.77-365.13)$ \\
\hline $\begin{array}{l}\text { Urinary CTLA-4 (ng/g } \\
\text { creat) }\end{array}$ & $151.70 \pm 27.01$ & $457.83 \pm 99.45$ & $299.53 \pm 47.46$ \\
\hline$(95 \% \mathrm{CI})$ & $(96.61-206.79)$ & $(251.58-664.08)$ & $(201.10-397.94)$ \\
\hline Serum CD80 (ng/l) & $0.87 \pm 0.12$ & $0.55 \pm 0.11$ & $0.95 \pm 0.22$ \\
\hline$(95 \% \mathrm{CI})$ & $(0.63-1.11)$ & $(0.32-0.78)$ & $(0.49-1.46)$ \\
\hline Serum CTLA-4 (ng/l) & $0.33 \pm 0.07$ & $0.45 \pm 0.12$ & $0.31 \pm 0.05$ \\
\hline$(95 \% \mathrm{CI})$ & $(0.19-0.47)$ & $(0.20-0.71)$ & $(0.20-0.42)$ \\
\hline \multicolumn{4}{|l|}{ Glucocorticoids treatments } \\
\hline None & 19 & 3 & 16 \\
\hline 1-7 days & 9 & 0 & 1 \\
\hline $8-28$ days & 1 & 0 & 4 \\
\hline 29-112 days & 0 & 6 & 5 \\
\hline $\begin{array}{l}\quad>112 \text { days or other } \\
\text { immunodepressive therapy }\end{array}$ & 3 & 14 & 0 \\
\hline
\end{tabular}

a Data are mean \pm s.e.m

$3 \quad$ b CI: Confidence Interval for mean 
Figure 1

Urinary CD80 and urinary CTLA-4 levels of steroid-sensitive MCD patients in relapse and remission
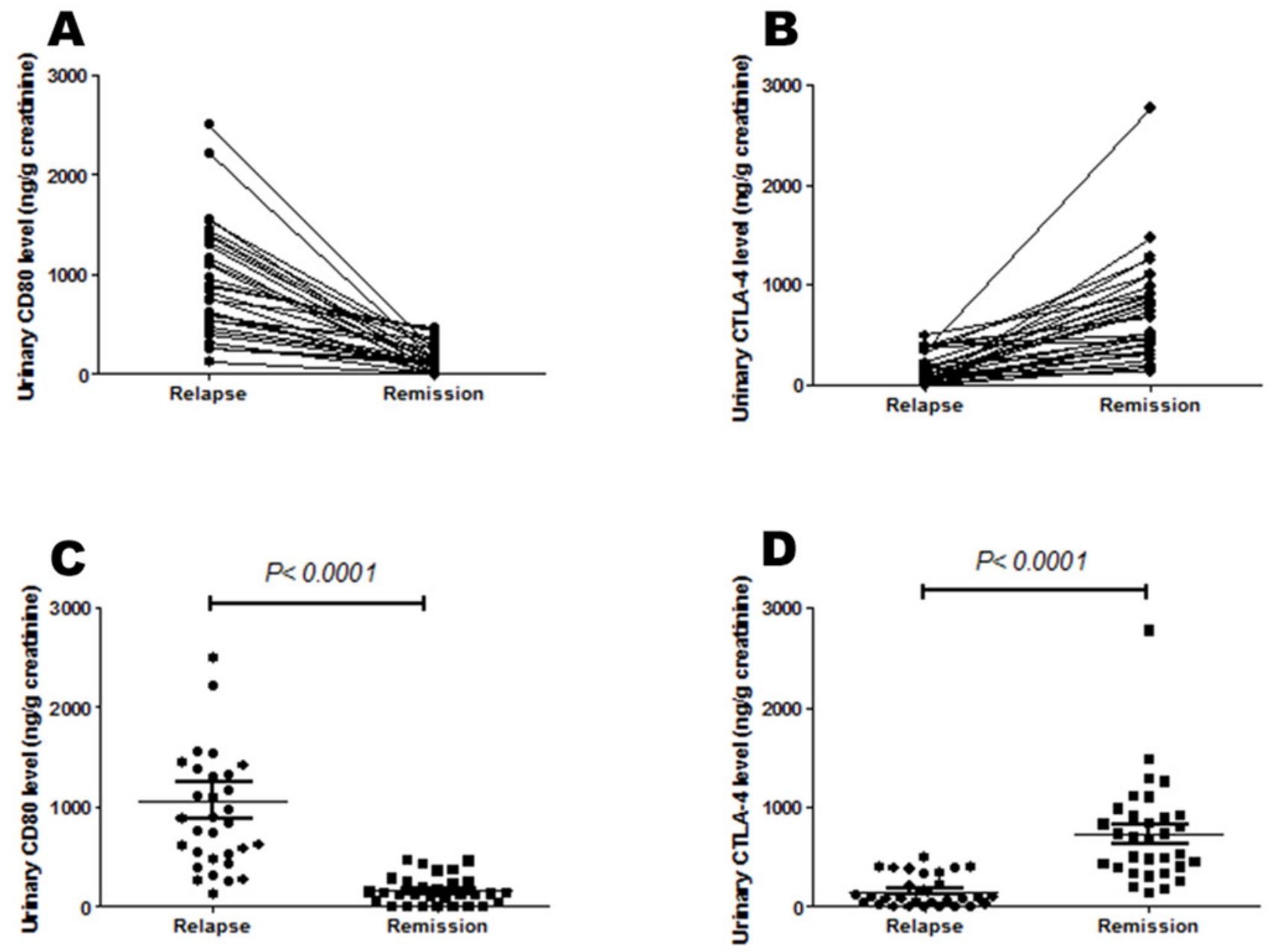
Figure 2

Receiver operating characteristic curves for differentiating relapse and remission in patients with steroid-sensitive MCD 


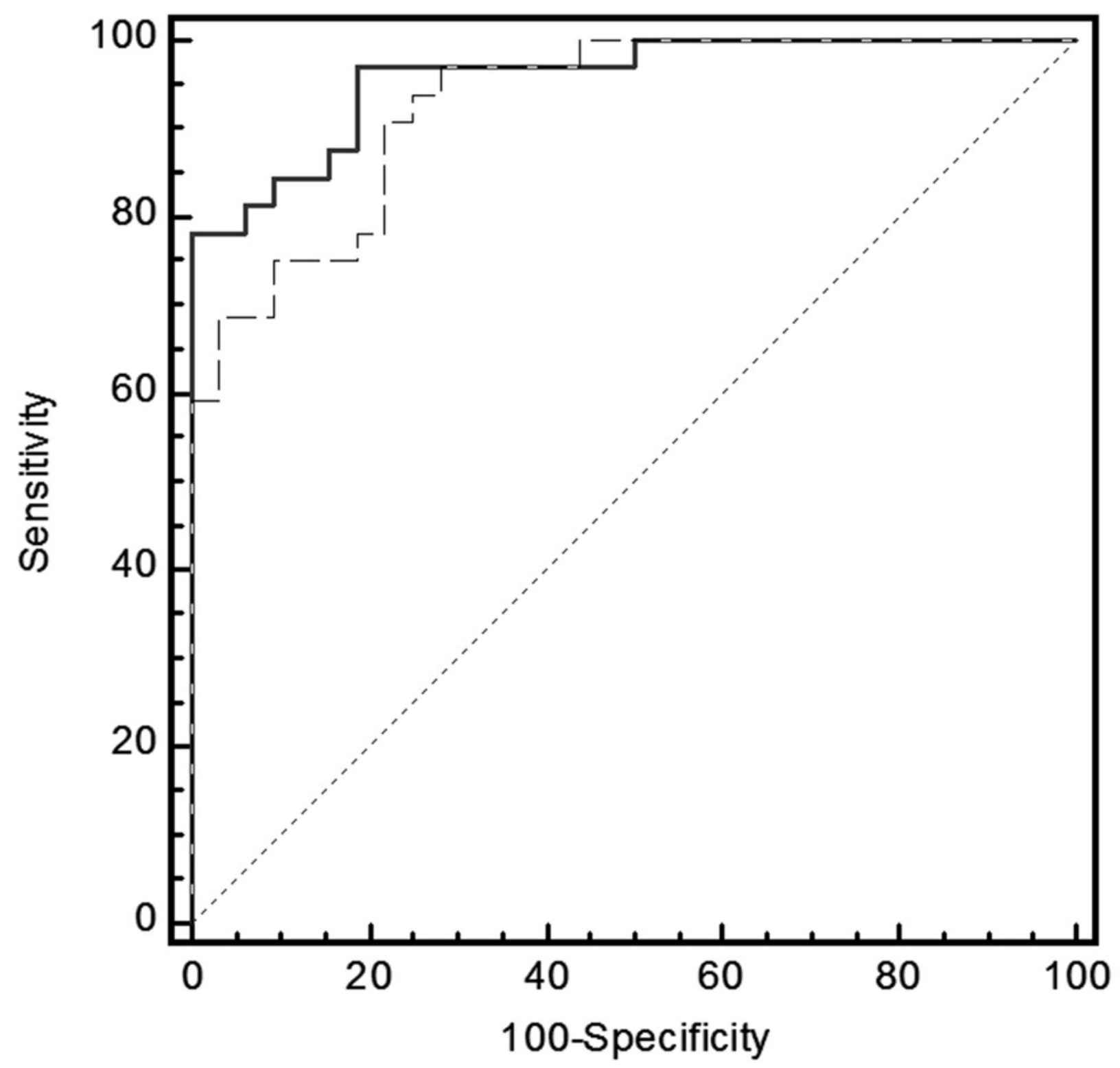

— Urinary CD80

- - Urinary CTLA-4

\begin{tabular}{|c|c|c|c|} 
& AUC & SE & \multicolumn{2}{c}{$95 \%$ CI } \\
\hline Urinary CD80 & 0.957 & 0.0265 & 0.875 to 0.992 \\
\hline Urinary CTLA-4 & 0.928 & 0.0343 & 0.835 to 0.977 \\
\hline
\end{tabular}


Figure 3

Comparison of urinary CD80 and CTLA-4 levels of steroid-sensitive MCD patients in relapse, steroid-resistant MCD patients in relapse and IMN in relapse
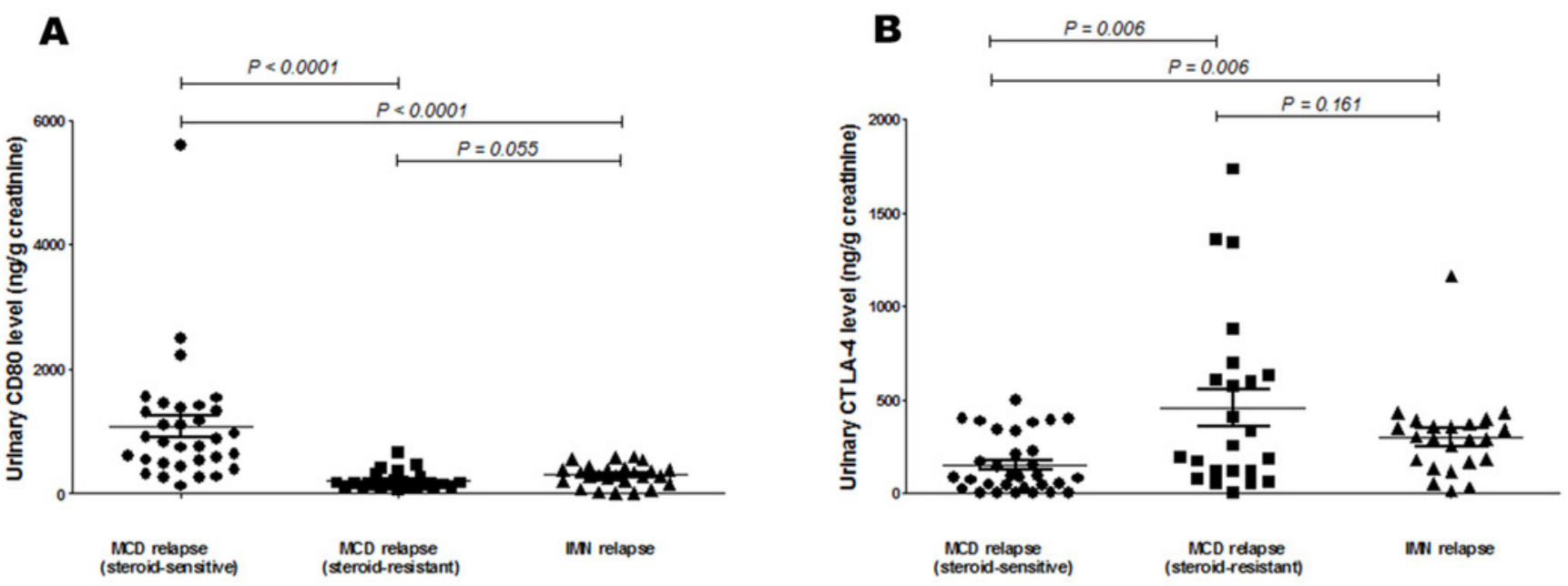
Figure 4

Receiver operating characteristic curves (ROC) for urinary CD80 with CTLA-4 levels differentiating patients with steroids-sensitive MCD and others.

ROC analysis of urinary CD80 with CTLA-4 levels comparing steroid-sensitive MCD and steroid- resistant MCD patients in relapse (a) and steroid-sensitive MCD and IMN patients in relapse (b).

A

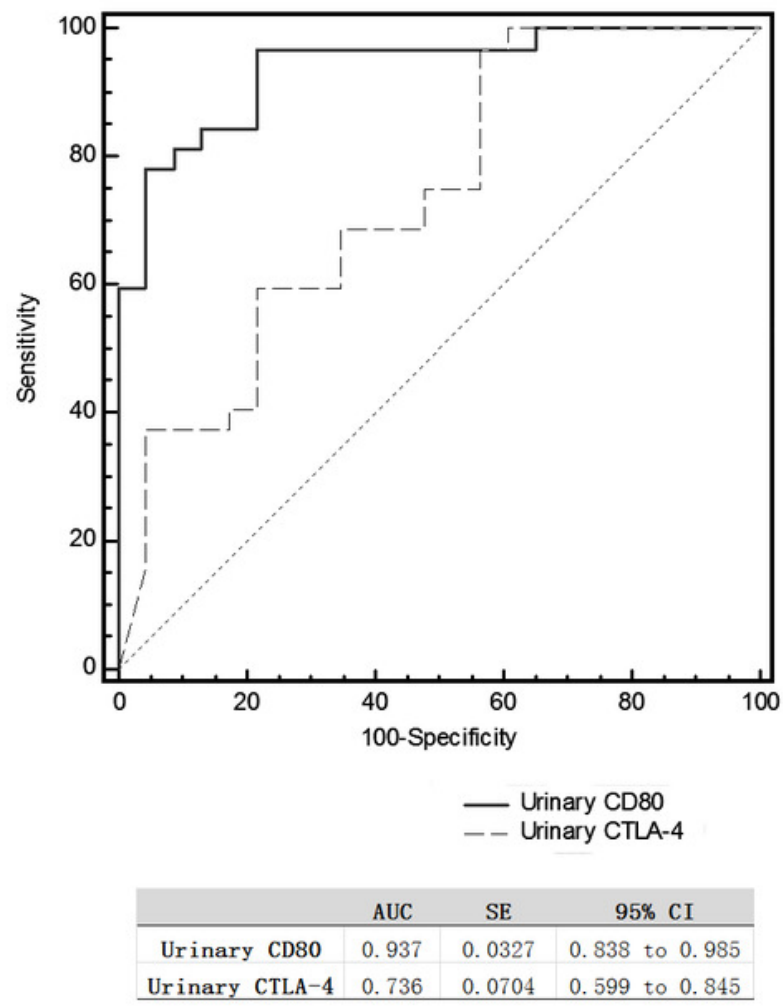

$\mathbf{B}$

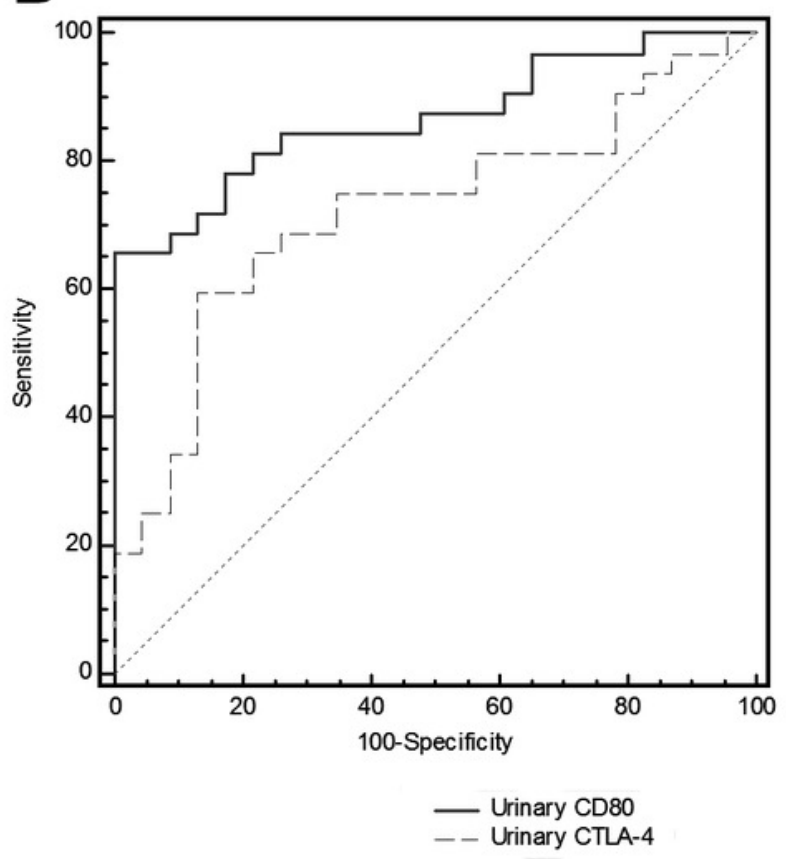

\begin{tabular}{|c|c|c|c|}
\hline & AUC & SE & $95 \%$ CI \\
\hline Urinary CD80 & 0.867 & 0.0483 & 0.748 to 0.943 \\
\hline Urinary CTLA-4 & 0.721 & 0.0716 & 0.584 to 0.834 \\
\hline
\end{tabular}




\section{Figure 5}

Expression of CD80 and CTLA-4 in glomerulus of several types of idiopathic nephrotic syndrome.

(a1, c1, and d1) CD80 was expressed (brown stain) in the glomerulus from a steroid-sensitive MCD patient in relapse, a steroid-resistant MCD patient in relapse, and an IMN patient in relapse. (b1) Minimal stain for CD80 was found in the glomerulus of a steroid sensitive MCD in partial remission. (b2, c2, and d2) CTLA-4 was expressed in the glomerulus from the steroid-resistant MCD patient in remission, the steroid-resistant MCD patient in relapse, and an IMN patient in relapse. (a2) CTLA-4 was absent in the glomerulus from the steroid sensitive MCD patient in relapse. (Immunohistochemistry, original magnification $\times 200$ ). 
CD80

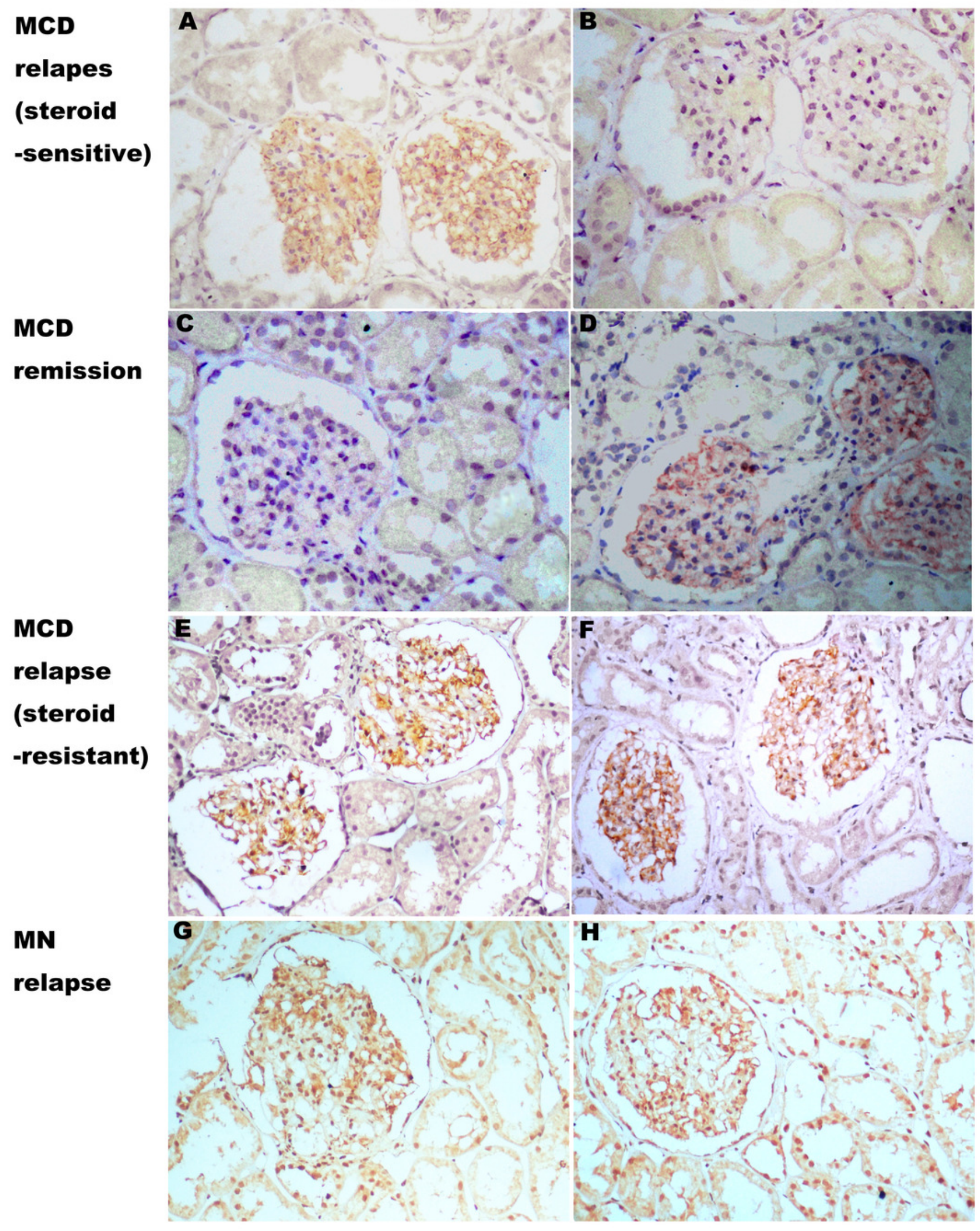

